\title{
Lorentz-violating contributions to the nuclear Schiff moment and nuclear EDM
}

\author{
Jonas B. Araujo, ${ }^{*}$ Rodolfo Casana, ${ }^{\dagger}$ and Manoel M. Ferreira, Jr. \\ Departamento de Física, Universidade Federal do Maranhão, \\ Campus Universitário do Bacanga, São Luís, Maranhão 65080-805, Brazil
}

(Received 19 November 2017; published 22 March 2018)

\begin{abstract}
In the context of an atom endowed with nuclear electric dipole moments (EDM), we consider the effects on the Schiff moment of $C P T$-even Lorentz-violating (LV) terms that modify the Coulomb potential. First, we study the modifications on the Schiff moment when the nucleus interacts with the electronic cloud by means of a Coulomb potential altered only by the $P$-even LV components. Next, by supposing the existence of an additional intrinsic LV EDM generated by other LV sources, we assess the corrections to the Schiff moment when the interaction nucleus-electrons runs mediated by a Coulomb potential modified by both the $P$-odd and $P$-even $\mathrm{LV}$ components. We then use known estimates and EDM measurements to discuss upper bounds on the new Schiff moment components and the possibility of a nuclear EDM component ascribed to LV effects.
\end{abstract}

DOI: 10.1103/PhysRevD.97.055032

\section{INTRODUCTION}

The possibility of detecting permanent electric dipole moments (EDMs) cannot be underestimated since it is related to a few major problems in contemporary physics [1-4]. The EDM interactions are $P$ odd and $T$ odd and, consequently, $C P$ odd (if the $C P T$ theorem holds). The breaking of $C P$ is one of the crucial Sakharov conditions for the baryon asymmetry in the Universe [5], and the CKM matrix is insufficient to account for it alone, so that there is room for new physics or some as-yet-unknown phenomenology from other sectors of the standard model. In the strong interactions, $C P$ violations are parametrized by the $\theta$ term, which is extremely small for as of yet unknown reasons. This poses the strong $C P$ problem, whose solution via spontaneous breaking of the Peccei-Quinn symmetry involves axions [6]. Although yet undetected, axions could induce oscillating EDMs [7].

The $P$ and $T$-odd nuclear forces could generate EDM by rendering charge fluctuations over a finite-sized nucleus. To date, the best experimental upper bound on a nuclear $\mathrm{EDM}$ is $\left|d\left({ }^{199} \mathrm{Hg}\right)\right|<7.4 \times 10^{-30} e \mathrm{~cm}$ [8]. According to

\footnotetext{
*jonas.araujo88@gmail.com rodolfo.casana@gmail.com *manojr.ufma@gmail.com
}

Published by the American Physical Society under the terms of the Creative Commons Attribution 4.0 International license. Further distribution of this work must maintain attribution to the author(s) and the published article's title, journal citation, and DOI. the Schiff theorem [9], in an atom with a pointlike nucleus and nonrelativistic electrons that interact only electrostatically, the nuclear EDM is completely screened at first order by the atom's electrons [9], causing no Stark spectrum shift. For a finite-sized nucleus, however, the first order screening is no longer complete, there appearing the nuclear Schiff moment, whose interaction with the electrons generates atomic EDM [9-11]. Such a nuclear EDM might yield an electric dipole moment for the atom as a whole by a process that involves the mixing of electron wave functions of opposite parity. The Schiff moment physics has been extensively investigated, with several discussions and corrections having been performed upon it. Experimental and theoretical proposals to verify considerable enhancements to the octupole and Schiff moments in heavy nuclei have been considered [12-15]. A proper relativistic treatment of the electrons in an atom with a finite-sized nucleus was considered in Ref. [16], with the generalization of the Schiff moment and the evaluation of the local dipole moment (LDM) incorporating the relativistic corrections. Numerical evaluations of the Schiff moment [17] and their relation to the atomic EDM magnitude (for a few heavy atoms) are also known [18]. Further developments include, for example, a more general form of the Schiff moment obtained via calculations at the operator level [19], the evaluation of internal nucleon contribution to the Schiff moment [20], the enhancement of Schiff and octupole moments (by more than 2 orders of magnitude) in atoms with asymmetrically deformed nuclei with collective $P$ and $T$-odd electromagnetic interactions [21], analysis of the Schiff theorem in ions and molecules [22], and other important results [23]. 
Lorentz-violating (LV) theories have been under investigation since the 1990s in different theoretical frameworks. In the standard model extension (SME) [24], many developments were performed in several sectors of interactions, yielding tight constraints on the magnitude of the violation coefficients [25]. In the gauge electromagnetic sector, many studies have scrutinized the effects of the $C P T$-odd [26] and $C P T$-even terms [27]. The $C P T$-even gauge photon sector is modified by the tensor $\left(K_{F}\right)^{\alpha \beta \mu \nu}[27,28]$, whose $P$-odd and $P$ even anisotropic repercussions on the Coulomb potential were properly evaluated [29-31]. Nuclear systems have also been a suitable environment to test and examine Lorentz violation, involving detailed analysis of beta decay [32] and nuclear spin/magnetic dipole moment calculations [33]. Recently, some implications of the $P$-even coefficients, $\left(k_{\mathrm{DE}}\right)^{i j}$, on the Coulomb potential were investigated in atoms with non-null nuclear quadrupole moments $\left(Q_{i j}\right)$. Estimating the energy anisotropy yielded by the interaction of the nuclear quadrupole moment with valence protons, $\delta E=K\left(k_{\mathrm{DE}}\right)^{i j} Q_{i j}$, very tight bounds were set on the LV parameters [34].

$\mathrm{LV}$ theories were also applied to address the muon magnetic dipole moment [35], the neutron EDM [36], one-loop contributions to lepton EDM induced by the a LV fermion term [37], and by the $C P T$-odd gauge coefficient [38], which has also been examined in other respects [39]. Some $C P T$-even coefficients, originally belonging to the tensor $\left(K_{F}\right)^{\alpha \beta \mu \nu}$, were also considered in nonminimal couplings between fermions and photons, with the focus on the EDM generation [40-42]. Nonminimal couplings involving higher derivatives $[43,44]$ and higher-dimension operators [45] were also considered. However, to date, no study has been proposed to investigate the impact of a spacetime anisotropy, stemming from Lorentz symmetry violation, on the EDM issues connected to the Schiff theorem or the nuclear Schiff moment. In the present paper, we evaluate the contributions that a Lorentz-violating anisotropic Coulomb potential may induce to the atomic EDM shielding problem. More specifically, we calculate how the modified Coulomb potential and an intrinsic nuclear EDM, coming from the $C P T$-even Lorentz-violating tensor, $\left(K_{F}\right)^{\alpha \beta \mu \nu}$, yields corrections to the residual interaction known as the Schiff moment.

\section{SCHIFF MOMENT IN A LORENTZ-VIOLATING ENVIRONMENT}

We consider an atomic nucleus whose charge density is $\rho(\boldsymbol{r})=\rho_{0}(\boldsymbol{r})+\delta \rho(\boldsymbol{r})$, where $\rho_{0}(r)$ corresponds to the spherically symmetric part, normalized to unity, $\int \rho_{0}(r) d^{3} r=Z e$, and the charge density fluctuations due to $P$ and $T$-odd nuclear interactions are encoded in $\delta \rho(r)$, as described in Refs. [10,21]. A neutral atom has an electronic cloud with $N=Z$ electrons; otherwise, one has an ion of $(N-Z)$ charge. The nuclear EDM arises from the $P$ and $T$-odd interactions, being given by

$$
\boldsymbol{d}=Z e \int \boldsymbol{r} \delta \rho(\boldsymbol{r}) d^{3} r
$$

Now we regard an anisotropic Lorentz-violating Coulomb potential studied in Ref. [31], which yields the following potential:

$$
A_{0}(r)=\frac{1}{4 \pi} \frac{q}{r}\left((1-n)+\kappa^{i j} \frac{r^{i} r^{j}}{2 r^{2}}\right)
$$

for a point particle of charge $q$, where $\kappa^{i j}=\left(k_{e_{-}}\right)^{i j}$ is a symmetric, parity-even, and traceless tensor, and $n=$ $\operatorname{tr}\left(k_{\mathrm{DE}}\right) / 3$, with $\left(k_{\mathrm{DE}}\right)^{i j}=-2\left(K_{F}\right)^{0 i 0 j}$ (see Ref. [27]). Our goal is to investigate how this modified potential could change the conclusions of Schiff's theorem [9] or contribute to the Schiff moment. The starting point is writing out the Hamiltonian for an atom in a region with an external field $\boldsymbol{E}_{0}$,

$$
H=K+V_{0(\mathrm{LV})}+V+U_{(\mathrm{LV})}+W,
$$

with $K$ representing the kinetic term,

$$
K=-\sum_{i}^{N} \frac{1}{2 m_{e}} \frac{\partial^{2}}{\partial \boldsymbol{R}_{i}^{2}}-\frac{1}{2 M} \frac{\partial^{2}}{\partial \boldsymbol{q}_{N}^{2}},
$$

while the electrostatic potential $(V)$ due to the external field is

$$
V=-\sum_{i}^{N}\left(-e \boldsymbol{R}_{i}\right) \cdot \boldsymbol{E}_{0}-Z e \boldsymbol{q}_{N} \cdot \boldsymbol{E}_{0}
$$

Here, $\quad W=-\boldsymbol{d} \cdot \boldsymbol{E}_{0}$ is the interaction between the nuclear EDM and the external field. Above, $\boldsymbol{R}_{i}$ and $\boldsymbol{q}_{N}$ correspond to the $i$ th electron's and the nucleus positions, respectively, while $\boldsymbol{r}$ is measured starting from the nucleus' center. The anisotropic electrostatic potential between the atomic components (electron-electron, electrons-nucleus), $V_{0(\mathrm{LV})}$, now receives contributions stemming from the Lorentz-violating potential (2), that is,

$$
\begin{aligned}
V_{0(\mathrm{LV})} & =e^{2} \sum_{i>j}^{N}\left[\frac{(1-n)}{\left|\boldsymbol{R}_{i}-\boldsymbol{R}_{j}\right|}+\kappa^{k l} \frac{\left(\boldsymbol{R}_{i}-\boldsymbol{R}_{j}\right)^{k}\left(\boldsymbol{R}_{i}-\boldsymbol{R}_{j}\right)^{l}}{2\left|\boldsymbol{R}_{i}-\boldsymbol{R}_{j}\right|^{3}}\right] \\
& -Z e^{2} \sum_{i}^{N} \int \rho_{0}(\boldsymbol{r})\left[\frac{(1-n)}{\left|\tilde{\boldsymbol{R}}_{i}-\boldsymbol{r}\right|}+\kappa^{k l} \frac{\left(\tilde{\boldsymbol{R}}_{i}-\boldsymbol{r}\right)^{k}\left(\tilde{\boldsymbol{R}}_{i}-\boldsymbol{r}\right)^{l}}{2\left|\tilde{\boldsymbol{R}}_{i}-\boldsymbol{r}\right|^{3}}\right] d^{3} r,
\end{aligned}
$$

where $\tilde{\boldsymbol{R}}_{i}=\left(\boldsymbol{R}_{i}-\boldsymbol{q}_{N}\right)$ gives the position of the $i$ th electron from the nucleus. The same holds for the electrostatic potential, $U_{(\mathrm{LV})}=U_{(\mathrm{is})}+U_{(\mathrm{an})}$, which accounts for the interaction of the $N$ electrons with the nuclear charge fluctuation, $\delta \rho(r)$, whose isotropic and anisotropic LV interactions, $U_{(\mathrm{is})}, U_{(\mathrm{an})}$, are written as 


$$
\begin{gathered}
U_{(\mathrm{is})}=-Z e^{2} \sum_{i}^{N} \int \delta \rho(\boldsymbol{r})(1-n) \frac{d^{3} r}{\left|\tilde{\boldsymbol{R}}_{i}-\boldsymbol{r}\right|}, \\
U_{(\mathrm{an})}=-Z e^{2} \sum_{i}^{N} \int \delta \rho(\boldsymbol{r})\left[\frac{\kappa^{k l}}{2} \frac{\left(\tilde{\boldsymbol{R}}_{i}-\boldsymbol{r}\right)^{k}\left(\tilde{\boldsymbol{R}}_{i}-\boldsymbol{r}\right)^{l}}{\left|\tilde{\boldsymbol{R}}_{i}-\boldsymbol{r}\right|^{3}}\right] d^{3} r,
\end{gathered}
$$

respectively. In order to investigate the possible contributions, we need to expand $U_{(\mathrm{LV})}$ in powers of $\left|\tilde{\boldsymbol{R}}_{i}\right|^{-1}$. In this task, there appear terms such as

$$
\begin{aligned}
\partial_{i} \partial_{j} \partial_{k}\left(\frac{1}{\tilde{R}}\right)= & {\left[\partial_{i} \partial_{j} \partial_{k}-\frac{1}{5}\left(\delta_{i j} \partial_{k}+\delta_{i k} \partial_{j}+\delta_{j k} \partial_{i}\right) \partial^{2}\right] \frac{1}{\tilde{R}} } \\
& +\frac{1}{5}\left(\delta_{i j} \partial_{k}+\delta_{i k} \partial_{j}+\delta_{j k} \partial_{i}\right) \partial^{2} \frac{1}{\tilde{R}}
\end{aligned}
$$

In accordance with the literature [10,15,22], the term inside brackets in Eq. (8) corresponds to an octupole moment operator that can be neglected. In addition, the charge octupole,

$$
o_{i j k}=r^{i} r^{j} r^{k}-\frac{r^{2}}{5}\left(\delta_{i j} r^{k}+\delta_{i k} r^{j}+\delta_{j k} r^{i}\right)
$$

can also be used to manipulate terms appearing in the expansions [21] and to further separate the ones in $o_{i j k}$. It just so happens that the usual Schiff moment calculation yields the same result for both procedures. This is not the case, however, when anisotropic pieces are involved. First, we expand its isotropic part (the first piece), $U_{\text {(is) }}$, as

$$
\begin{aligned}
U_{(\mathrm{is})} \approx & (1-n)\left\{-e \boldsymbol{d} \cdot \sum_{i}^{N} \frac{\tilde{\boldsymbol{R}}_{i}}{\left|\tilde{\boldsymbol{R}}_{i}\right|^{3}}\right. \\
& \left.-4 \pi \frac{Z e^{2}}{10} \int d^{3} r \delta \rho(\boldsymbol{r}) r^{2} \boldsymbol{r} \cdot \sum_{i}^{N} \nabla_{i}\left[\delta\left(\tilde{\boldsymbol{R}}_{i}\right)\right]\right\} .
\end{aligned}
$$

In order to expand the anisotropic part, we need to write $a=\left(\tilde{\boldsymbol{R}}_{i}-\boldsymbol{r}\right)^{k}\left(\tilde{\boldsymbol{R}}_{i}-\boldsymbol{r}\right)^{l} /\left|\tilde{\boldsymbol{R}}_{i}-\boldsymbol{r}\right|^{3}$, as a Taylor series, that is,

$$
\begin{aligned}
a \approx & -r^{m} \partial_{m}\left[\frac{\left(\tilde{\boldsymbol{R}}_{i}\right)^{k}\left(\tilde{\boldsymbol{R}}_{i}\right)^{l}}{\left|\tilde{\boldsymbol{R}}_{i}\right|^{3}}\right]+\frac{1}{6} r^{m} r^{p} r^{q} \\
& \times\left[\partial_{k} \partial_{m} \partial_{p} \partial_{q}\left(\frac{1}{\left|\tilde{\boldsymbol{R}}_{i}\right|}\right)\left(\tilde{\boldsymbol{R}}_{i}\right)^{l}+\partial_{p} \partial_{q} \partial_{k}\left(\frac{1}{\left|\tilde{\boldsymbol{R}}_{i}\right|}\right) \delta_{m l}\right. \\
& \left.+\partial_{m} \partial_{q} \partial_{k}\left(\frac{1}{\left|\tilde{\boldsymbol{R}}_{i}\right|}\right) \delta_{p l}+\partial_{m} \partial_{p} \partial_{k}\left(\frac{1}{\left|\tilde{\boldsymbol{R}}_{i}\right|}\right) \delta_{q l}\right],
\end{aligned}
$$

where we omitted the zeroth and second order terms, for $\delta \rho(\boldsymbol{r})$ is odd. We have also used the fact that

$$
\frac{\left(\tilde{\boldsymbol{R}}_{i}\right)^{k}\left(\tilde{\boldsymbol{R}}_{i}\right)^{l}}{\left|\tilde{\boldsymbol{R}}_{i}\right|^{3}}=-\partial_{k}\left(\frac{1}{\left|\tilde{\boldsymbol{R}}_{i}\right|}\right)\left(\tilde{\boldsymbol{R}}_{i}\right)^{l}
$$

Note, still, that the term

$$
r^{m} r^{p} r^{q} \partial_{k} \partial_{m} \partial_{p} \partial_{q}\left(\frac{1}{\left|\tilde{\boldsymbol{R}}_{i}\right|}\right)\left(\tilde{\boldsymbol{R}}_{i}\right)^{l},
$$

in Eq. (11), can only be unambiguously rewritten by grouping $r^{m} r^{p} r^{q}$ as in Eq. (9), in which we ignore the charge octupole term, $o_{i j k}$, as usual. After this step, this ambiguity is removed, and we can use (8) to address the remaining derivatives. This procedure enables us to correctly expand the anisotropic term, achieving the full expression

$$
\begin{aligned}
U_{(\mathrm{LV})} \approx & (1-n)\left\{-e \boldsymbol{d} \cdot \sum_{i}^{N} \frac{\tilde{\boldsymbol{R}}_{i}}{\left|\tilde{\boldsymbol{R}}_{i}\right|^{3}}\right. \\
& \left.-4 \pi \frac{Z e^{2}}{10} \int d^{3} r \delta \rho(\boldsymbol{r}) r^{2} \boldsymbol{r} \cdot \sum_{i}^{N} \nabla_{i}\left[\delta\left(\tilde{\boldsymbol{R}}_{i}\right)\right]\right\} \\
& +e \sum_{i}^{N} \frac{(\kappa)^{k l}}{2} d^{m} \partial_{m}\left[\frac{\left(\tilde{\boldsymbol{R}}_{i}\right)^{k}\left(\tilde{\boldsymbol{R}}_{i}\right)^{l}}{\left|\tilde{\boldsymbol{R}}_{i}\right|^{3}}\right] \\
& +4 \pi \sum_{i}^{N} \int r^{2} \delta \rho(\boldsymbol{r}) \frac{\kappa^{k l}}{2} \frac{Z e^{2}}{10} \\
& \times\left[\left(\tilde{\boldsymbol{R}}_{i}\right)^{l} r^{i} \partial_{i} \partial_{k}+\frac{2}{5} \delta_{k l} r^{i} \partial_{i}+\frac{9}{5} r^{l} \partial_{k}\right] d^{3} r \delta\left(\tilde{\boldsymbol{R}}_{i}\right) .
\end{aligned}
$$

Now it is necessary to introduce the displacement operator

$$
Q_{\mathrm{D}}=\frac{\boldsymbol{d}}{Z e} \cdot \frac{\partial}{\partial \boldsymbol{q}_{N}}
$$

which allows us to compute the commutator $\left[Q_{\mathrm{D}}, V_{0(\mathrm{LV})}\right]=$ $\frac{d}{Z e} \cdot \frac{\partial}{\partial \boldsymbol{q}_{N}}\left[V_{0(\mathrm{LV})}\right]$. Deriving with respect to the coordinates $\boldsymbol{q}_{N}^{l}$ is equivalent to deriving in the components $\left(\boldsymbol{R}_{i}\right)^{l}$, since $\partial_{l\left(\boldsymbol{q}_{N}\right)} F\left(\boldsymbol{R}_{i}-\boldsymbol{q}_{N}\right)=-\partial_{l\left(\boldsymbol{R}_{i}\right)} F\left(\boldsymbol{R}_{i}-\boldsymbol{q}_{N}\right)$, or simply $\partial / \partial \boldsymbol{q}_{N}=$ $-\partial / \partial \boldsymbol{R}_{i}$. Expanding the derivatives $\partial V_{0(\mathrm{LV})} / \partial \boldsymbol{R}_{i}$ [in a similar way to the ones performed on $\left.U_{(\mathrm{LV})}\right]$, making simplifications, the following commutator is obtained,

$$
\begin{aligned}
{\left[Q_{\mathrm{D}}, V_{0(\mathrm{LV})}\right] } \\
=U_{(\mathrm{LV})}-4 \pi e \sum_{i}^{N} \frac{1}{10} \\
\quad \times\left\{Z e \int d^{3} r \delta \rho(\boldsymbol{r}) r^{2}\left[(1-n) r^{k}-\frac{9}{10} r^{l} \kappa^{k l}\right]\right. \\
\left.\quad-\frac{5}{3} \int r^{2} \rho_{0}(\boldsymbol{r}) d^{3} r\left[(1-n) d^{k}-\frac{2}{5} d^{l} \kappa^{k l}\right]\right\} \partial_{k} \delta\left(\tilde{\boldsymbol{R}}_{i}\right),
\end{aligned}
$$

where $U_{(\mathrm{LV})}$ is given by Eq. (14) and $\partial_{k} \delta\left(\tilde{\boldsymbol{R}}_{i}\right)=$ $\partial_{k\left(\boldsymbol{R}_{i}-\boldsymbol{q}_{N}\right)}\left[\delta\left(\boldsymbol{R}_{i}-\boldsymbol{q}_{N}\right)\right]$. This calculation implies that $\left[Q_{\mathrm{D}}, V_{0(\mathrm{LV})}\right]=U_{(\mathrm{LV})}-H_{\text {residual }}$. On the other hand, $\left[Q_{\mathrm{D}}, V\right]=W,\left[Q_{\mathrm{D}}, K\right]=0$, so that the full Hamiltonian (3) can be written as 


$$
H=H_{0(\mathrm{LV})}+\left[Q_{\mathrm{D}}, H_{0(\mathrm{LV})}\right]+H_{\text {residual }},
$$

with $H_{0(\mathrm{LV})}=K+V_{0(\mathrm{LV})}+V$ and $U_{(\mathrm{LV})}+W=\left[Q_{\mathrm{D}}, H_{0(\mathrm{LV})}\right]-$ $H_{\text {residual }}$. Equation (17) implies the shielding of the pointlike dipole except for the residual interaction, consequence of the finite nuclear size, which provides the atom with EDM. In this case, the atomic EDM will be generated by a Schiff-like moment that appears inside the residual interaction term, $H_{\text {residual }}=H_{\text {Schiff (LV) }}$, read from Eq. (16) as

$$
H_{\text {Schiff }(\mathrm{LV})}=-4 \pi e \mathbb{S}_{(1)}^{k} \sum_{i}^{N} \partial_{k} \delta\left(\tilde{\boldsymbol{R}}_{i}\right),
$$

where the modified Schiff moment,

$$
\mathbb{S}_{(1)}^{k}=(1-n) S^{k}-S_{(\mathrm{LV} 1)}^{k},
$$

is composed of two contributions,

$S^{k}=\frac{1}{10}\left(Z e \int \delta \rho(\boldsymbol{r}) r^{2} r^{k} d^{3} r-\frac{5}{3} d^{k} \int r^{2} \rho_{0}(\boldsymbol{r}) d^{3} r\right)$,

$S_{(\mathrm{LV} 1)}^{k}=\frac{\kappa^{k l}}{10}\left(Z e \frac{9}{10} \int \delta \rho(\boldsymbol{r}) r^{2} r^{l} d^{3} r-\frac{2}{3} d^{l} \int r^{2} \rho_{0}(\boldsymbol{r}) d^{3} r\right)$.

The usual Schiff moment, $S^{k}$, is induced by the $P$ and $T$-odd interactions that generate the charge fluctuations $\delta \rho(r)$, while $S_{(\mathrm{LV} 1)}^{k}$ is the anisotropic piece associated with the LV tensor, $\kappa^{k l}$. The Lorentz violation also contributes by yielding the factor $(1-n)$, which alters the weight of the usual moment $\left(S^{k}\right)$ on the total modified Schiff moment. Although the LV term containing $\kappa^{k l}$ does not act as source of elementary dipole moment, $\boldsymbol{d}$, it creates a new Schiff moment component, which impacts the atomic electric dipole moment in an anisotropic manner. In writing $S_{(\mathrm{LV} 1)}^{k}=\kappa^{k l} \tilde{S}^{l}$, with

$\tilde{S}^{l}=\frac{1}{10}\left[Z e \frac{9}{10} \int \delta \rho(\boldsymbol{r}) r^{2} r^{l} d^{3} r-\frac{2}{3} d^{l} \int r^{2} \rho_{0}(\boldsymbol{r}) d^{3} r\right]$,

and comparing the expressions (20) and (21), one notes that the LV kernel, $\tilde{S}^{l}$, has in principle the same order of magnitude and the same direction as the usual Schiff moment, $S^{k}$.

A point worth mentioning is that the usual Schiff moment is aligned with the nuclear spin (I), that is, $\boldsymbol{S}=S \hat{\mathbf{I}}$. The same holds for $\tilde{\mathbf{S}}$. In contrast, the Lorentzviolating piece (21) is no longer aligned with the spin, for it is rotated by the matrix $\kappa^{k l}$, so that

$$
\mathbb{S}_{(1)}^{k}=S \hat{\mathbf{I}}^{k}+\tilde{S}\left(\kappa^{k l} \hat{\mathbf{I}}^{l}\right) .
$$

We note the existence of an EDM associated with the LV piece, $S_{(\mathrm{LV} 1)}^{k}$, which we represent as $\left(\mathbf{d}_{\mathrm{LV}}\right)^{k}=\alpha \kappa^{k l} \mathbf{d}^{l}$, being $\mathbf{d}^{l}$ the EDM associated with the usual Schiff moment and $\alpha$ a constant. Concerning the measurement respects, it is worth to discuss how the Schiff moment components not aligned with the spin could become manifest in usual experiments designed to detect nuclear EDM of atoms. In typical setups, an atom endowed with spin $\mathbf{I}$, magnetic moment $\boldsymbol{\mu}=\mu \mathbf{I}$ and EDM $\mathbf{d}=d \mathbf{I}$, is placed in a region with an electric and a magnetic field, which cause a kind of Zeeman interaction, $U=-(\mu \mathbf{B}+d \mathbf{E}) \cdot \mathbf{I}$. This interaction implies precession frequency around the $\boldsymbol{B}$ axis equal to $\omega_{i}=(\mu B+d E) / 2$ (for spin $1 / 2$ systems). These experiments begin with parallel $\boldsymbol{E}$ and $\boldsymbol{B}$ fields, then the electric field is inverted, modifying the precession frequency to $\omega_{f}=(\mu B-d E) / 2$. The measured precession variation is a response due solely to the EDM, $\Delta \omega=d E$. In principle, the fact that the LV piece $S_{(\mathrm{LV} 1)}^{k}$ is not parallel to the spin does not avoid its detection, since the spin, even if initially prepared in one particular axis, will precess around the magnetic field direction. The point is that the LV EDM piece will yield precession around the magnetic field as well, inasmuch as it also is written in terms of the nuclear spin. It implies a variation on the frequency precession, $\Delta \omega_{L V}=\left|\mathbf{d}_{L V}\right| E$, after inverting the electric field. The key experimental point is to find a way in which this new contribution could be separated from the usual one.

\section{INTRINSIC LV NUCLEAR EDM}

The $P$ and $T$-odd interactions act as possible generators of nuclear intrinsic EDM in the atom. In a $C P T$-even scenario, the EDM interactions are also $C P$ odd; however, they can be $C P$ even in a $\mathrm{LV}$ and $C P T$-odd framework. It is worth supposing other sources (beyond the usual nuclear interactions) that could yield intrinsic EDM to the nucleus, not necessarily associated to the nuclear spin. This can be performed by Lorentz-violating $P$ and $T$-odd terms belonging to the $C P T$-even and $C P T$-odd quark sector of the SME [24]. It can be also triggered by dimension-five $C P T$-odd and $C P$ even nonminimal interactions between quarks and the electromagnetic field [46]. Once the intrinsic nuclear $\operatorname{LV} \operatorname{EDM}\left(\boldsymbol{d}_{\text {ilv }}\right)$ is generated, it is uniformly distributed over the nucleus and interacts with the electronic cloud by means of a modified Coulomb potential, as the one of Eq. (2). Now, we examine this situation, rewriting $U_{(\text {is) }}$ and $U_{(\text {an) }}$ in Eqs. (6), (7), as

$$
\begin{aligned}
U_{(\mathrm{is})}^{\prime}= & -e(1-n) \boldsymbol{d}_{\mathrm{ilv}} \cdot \sum_{i}^{N} \frac{\tilde{\boldsymbol{R}}_{i}}{\left|\tilde{\boldsymbol{R}}_{i}\right|^{3}} \\
& -Z e^{2} \sum_{i}^{N} \int \delta \rho(\boldsymbol{r})(1-n) \frac{d^{3} r}{\left|\tilde{\boldsymbol{R}}_{i}-\boldsymbol{r}\right|},
\end{aligned}
$$




$$
\begin{aligned}
U_{(\mathrm{an})}^{\prime}= & e \frac{\kappa^{k l}}{2} \sum_{i}^{N}\left[-3 \frac{\left(\tilde{\boldsymbol{R}}_{i}\right)^{k}\left(\tilde{\boldsymbol{R}}_{i}\right)^{l} \boldsymbol{d}_{\mathrm{ilv}} \cdot \tilde{\boldsymbol{R}}_{i}}{\left|\tilde{\boldsymbol{R}}_{i}\right|^{5}}+2 \frac{\boldsymbol{d}_{\mathrm{ilv}} \cdot \tilde{\boldsymbol{R}}_{i}}{\left|\tilde{\boldsymbol{R}}_{i}\right|^{3}}\right] \\
& -Z e^{2} \sum_{i}^{N} \int \delta \rho(\boldsymbol{r})\left[\frac{\kappa^{k l}}{2} \frac{\left(\tilde{\boldsymbol{R}}_{i}-\boldsymbol{r}\right)^{k}\left(\tilde{\boldsymbol{R}}_{i}-\boldsymbol{r}\right)^{l}}{\left|\tilde{\boldsymbol{R}}_{i}-\boldsymbol{r}\right|^{3}}\right] d^{3} r .
\end{aligned}
$$

The new potential $\mathbb{U}^{\prime}=U_{(\text {is })}^{\prime}+U_{(\text {an })}^{\prime}$ is expanded as before, yielding

$$
\begin{aligned}
U_{(\mathrm{LV} 2)} \approx & (1-n)\left\{-e\left(\boldsymbol{d}_{\mathrm{ilv}}+\boldsymbol{d}_{\delta}\right) \cdot \sum_{i}^{N} \frac{\tilde{\boldsymbol{R}}_{i}}{\left|\tilde{\boldsymbol{R}}_{i}\right|^{3}}\right. \\
& \left.-4 \pi \frac{Z e^{2}}{10} \int d^{3} r \delta \rho(\boldsymbol{r}) r^{2} \boldsymbol{r} \cdot \sum_{i}^{N} \nabla_{i}\left[\delta\left(\tilde{\boldsymbol{R}}_{i}\right)\right]\right\} \\
& +e \sum_{i}^{N} \frac{\kappa^{k l}}{2}\left(\boldsymbol{d}_{\mathrm{int}}+\boldsymbol{d}_{\delta}\right)^{m} \partial_{m}\left[\frac{\left(\tilde{\boldsymbol{R}}_{i}\right)^{k}\left(\tilde{\boldsymbol{R}}_{i}\right)^{l}}{\left|\tilde{\boldsymbol{R}}_{i}\right|^{3}}\right] \\
& +4 \pi \sum_{i}^{N} \int r^{2} \delta \rho(\boldsymbol{r}) \frac{\kappa^{k l}}{2} \frac{Z e^{2}}{10} \\
& \times\left[\left(\tilde{\boldsymbol{R}}_{i}\right)^{l} r^{i} \partial_{i} \partial_{k}+\frac{2}{5} \delta_{k l} r^{i} \partial_{i}+\frac{9}{5} r^{l} \partial_{k}\right] d^{3} r \delta\left(\tilde{\boldsymbol{R}}_{i}\right),
\end{aligned}
$$

in which $\boldsymbol{d}_{\delta}$ stands for the EDM generated by the charge fluctuations. The displacement operator (15) is now rewritten as

$$
Q_{\mathrm{D}}=\frac{\left(\boldsymbol{d}_{\mathrm{ilv}}+\boldsymbol{d}_{\delta}\right)}{Z e} \cdot \frac{\partial}{\partial \boldsymbol{q}_{N}},
$$

leading to the following commutator:

$$
\begin{aligned}
{\left[Q_{\mathrm{D}}, V_{0(\mathrm{LV} 2)}\right]=} & U_{(\mathrm{LV} 2)}-4 \pi e \sum_{i}^{N} \frac{1}{10} \\
& \times\left\{Z e \int d^{3} r \delta \rho(\boldsymbol{r}) r^{2}\left[(1-n) r^{k}-\frac{9}{10} r^{l} \kappa^{k l}\right]\right. \\
& -\frac{5}{3} \int r^{2} \rho_{0}(\boldsymbol{r}) d^{3} r\left[(1-n)\left(\boldsymbol{d}_{\mathrm{ilv}}+\boldsymbol{d}_{\delta}\right)^{k}\right. \\
& \left.\left.-\frac{2}{5}\left(\boldsymbol{d}_{\mathrm{ilv}}+\boldsymbol{d}_{\delta}\right)^{p} \kappa^{k p}\right]\right\} \partial_{k} \delta\left(\tilde{\boldsymbol{R}}_{i}\right) .
\end{aligned}
$$

In comparison with Eq. (16), one notes that the total EDM is modified, $\boldsymbol{d} \rightarrow \boldsymbol{d}_{\text {ilv }}+\boldsymbol{d}_{\delta}$. Furthermore, the commutator $\left[Q_{\mathrm{D}}, V\right]$ still yields $W$, so that the Schiff shielding is preserved at first order, and the residual interaction keeps its form, $H_{\text {Schiff }(\mathrm{LV})}=-4 \pi e \mathbb{S}_{(2)}^{k} \sum_{i}^{N} \partial_{k} \delta\left(\tilde{\boldsymbol{R}}_{i}\right)$, with

$$
\mathbb{S}_{(2)}^{k}=(1-n) S_{(2)}^{k}+S_{(\mathrm{LV} 2)}^{k},
$$

being the modified Schiff moment, which now receives contributions from $\boldsymbol{d}_{\mathrm{ilv}}$ and $\boldsymbol{d}_{\delta}$, written in two pieces,

$$
\begin{aligned}
S_{(2)}^{k}= & \frac{1}{10}\left[\mathrm{Ze} \int \delta \rho(\boldsymbol{r}) r^{2} r^{k} d^{3} r\right. \\
& \left.-\frac{5}{3}\left(\boldsymbol{d}_{\mathrm{ilv}}+\boldsymbol{d}_{\delta}\right)^{k} \int r^{2} \rho_{0}(\boldsymbol{r}) d^{3} r\right], \\
S_{(\mathrm{LV} 2)}^{k}= & -\frac{\kappa^{k l}}{10}\left\{Z e \frac{9}{10} \int \delta \rho(\boldsymbol{r}) r^{2} r^{l} d^{3} r\right. \\
& \left.-\frac{2}{3}\left(\boldsymbol{d}_{\mathrm{ilv}}+\boldsymbol{d}_{\delta}\right)^{l} \int r^{2} \rho_{0}(\boldsymbol{r}) d^{3} r\right\} .
\end{aligned}
$$

We can rewrite (29) at first order in terms of the usual Schiff moment

$\mathbb{S}_{(2)}^{k}=(1-n) S^{k}-\frac{1}{6}\left(\boldsymbol{d}_{\mathrm{ilv}}\right)^{k} \int r^{2} \rho_{0}(\boldsymbol{r}) d^{3} r+S_{(\mathrm{LV} 1)}^{k}$,

where $S^{k}$ is the usual Schiff moment (20). Thus, in the presence of an intrinsic EDM, the Schiff theorem is kept unharmed, with the Schiff moment receiving a new contribution stemming from the intrinsic EDM. Note that the modified Schiff moment (32) generally does not point in the same direction as the nuclear spin $\mathbf{I}$, since the intrinsic moment $\boldsymbol{d}_{\text {ilv }}$ carries LV "rotations". At first order, one can neglect $\boldsymbol{d}_{\text {ilv }}$ inside $S_{(\mathrm{LV} 2)}^{k}$, given that $\boldsymbol{d}_{\text {ilv }}$ should also depend on LV coefficients, as commented in the Conclusions. For this reason, the LV Schiff moment (31) becomes equal to the one in Eq. (21).

In the following, we consider the case in which the total nuclear EDM, $\boldsymbol{d}_{\mathrm{ilv}}+\boldsymbol{d}_{\delta}$, interacts with the electronic cloud by a Coulomb potential altered by $P$-odd coefficients belonging to the tensor $\left(K_{F}\right)^{\alpha \beta \mu \nu}$, as $\kappa^{j}=\frac{1}{2} \epsilon^{j p q}\left(\kappa_{D B}\right)^{p q}$, with $\left(\kappa_{D B}\right)^{j k}=$ $\epsilon^{k p q}\left(K_{F}\right)^{0 j p q}$ [27]. In Ref. [30], the induced modifications to the Coulomb potential were evaluated at second order in $\kappa^{i}: A_{0}(\mathbf{r})=(q / 4 \pi)\left\{\left(1+c_{\kappa}\right) / r-(\boldsymbol{\kappa} \cdot \mathbf{r})^{2} / 2 r^{3}\right\}$, where $c_{\kappa}=-\kappa^{2} / 2$, in which the anisotropy appears at second order. Following the same steps, the modified Schiff moment is $\mathbb{S}_{(3)}^{k}=\left(1+c_{\kappa}\right) S_{(3)}^{k}-S_{(\mathrm{LV} 3)}^{k}$, which now receives contributions from $\boldsymbol{d}_{\mathrm{ilv}}$ and $\boldsymbol{d}_{\delta}$, written in two pieces,

$$
\begin{aligned}
S_{(3)}^{k}= & \frac{1}{10}\left[Z e \int \delta \rho(\boldsymbol{r}) r^{2} r^{k} d^{3} r\right. \\
& \left.-\frac{5}{3}\left(\boldsymbol{d}_{\mathrm{ilv}}+\boldsymbol{d}_{\delta}\right)^{k} \int r^{2} \rho_{0}(\boldsymbol{r}) d^{3} r\right], \\
S_{(\mathrm{LV} 3)}^{k}= & -\frac{\kappa^{k} \kappa^{l}}{10}\left\{Z e \frac{9}{10} \int \delta \rho(\boldsymbol{r}) r^{2} r^{l} d^{3} r\right. \\
& \left.-\frac{2}{3}\left(\boldsymbol{d}_{\mathrm{ilv}}+\boldsymbol{d}_{\delta}\right)^{l} \int r^{2} \rho_{0}(\boldsymbol{r}) d^{3} r\right\},
\end{aligned}
$$


which, at first order in the $\mathrm{LV}$ parameter $\left(\kappa^{l}\right)$, is

$$
\mathbb{S}_{(3)}^{k}=S^{k}-\frac{1}{6}\left(\boldsymbol{d}_{\mathrm{ilv}}\right)^{k} \int r^{2} \rho_{0}(\boldsymbol{r}) d^{3} r
$$

\section{CONCLUSIONS AND FINAL REMARKS}

We have discussed the repercussions of LV terms belonging to the electromagnetic $C P T$-even sector of the SME to the nuclear Schiff moment and also the effect of an additional intrinsic LV nuclear EDM, $\boldsymbol{d}_{\mathrm{ilv}}$. The achieved modified LV Schiff moments (19), (32), (35) do not point in the same direction as the nuclear spin, as it occurs with the usual Schiff moment. Thus, it is important to examine how these LV components can be detected, regarding the small magnitude of all EDM pieces. In typical experiments to measure EDM, the atom spin $\mathbf{I}$ is prepared in a given direction, and then it interacts with controlled electric and magnetic fields, as already mentioned. A way to isolate the LV contribution could be preparing the nuclear spin state to point in the same direction as the magnetic field axis, $\mathbf{I}=|\mathbf{I}| \hat{z}$, with $\left|s_{z} \pm\right\rangle$. In this configuration, only the LV EDM component will yield torque and precession. The $x$ component of the Schiff moment (23), therefore, is given entirely in terms of $S_{(\mathrm{LV} 1)}^{k}$, that is,

$$
\mathbb{S}_{(1)}^{x}=\tilde{S}\left(\kappa^{13} \hat{\mathbf{I}}^{z}\right)
$$

so that the precession motion will be caused only by the $\kappa^{13} \tilde{S}^{3}$ component. In such a situation, usual EDM experiments can be used to constrain the magnitude of LV Schiff moment. Using recent calculations and experimental data, we can set bounds on the LV Schiff moment components, observing that $\tilde{S}$ and $S$ have the same order of magnitude. In Ref. [18], the EDM of a few atoms was estimated in terms of their Schiff moments. Among them, the ${ }^{199} \mathrm{Hg}$ atom is the one which possesses the most precise EDM measurement. According to those calculations, the magnitude of the EDM is $d\left({ }^{199} \mathrm{Hg}\right)=-2.8 \times 10^{-17}\left(S / e \mathrm{fm}^{3}\right) e \mathrm{~cm}$. The experimental data of Ref. [8] for the ${ }^{199} \mathrm{Hg} \mathrm{EDM}$ is, $\left|d\left({ }^{199} \mathrm{Hg}\right)\right|<7.4 \times 10^{-30} e \mathrm{~cm}$. In a setup as proposed above, the following upper bound is obtained:

$$
\left|\left\langle\mathbb{S}_{(1)}^{x}\right\rangle_{z \pm}\right|=\left|\tilde{S} \kappa^{13}\right| \lesssim 2.6 \times 10^{-13} e \mathrm{fm}^{3} .
$$

It is also relevant to point out that these bounds are set in the Earth's reference frame (RF), where the experimental apparatus is located, and that the LV coefficients are not constant in it. In order to write these results in terms of the coefficients measured in a Sun-based RF, where these coefficients are approximately constant, it is necessary to perform the sidereal analysis, i.e., translate the bounds between these RFs. We will consider, as in the literature $[41,47]$, the Earthbased lab's RF at the colatitude $\chi$, rotating around the Earth's axis with angular velocity $\Omega=2 \pi / 23 \mathrm{~h} 56$ s. For experiments up to a few weeks long, the transformation law for a rank-2 tensor is $A_{i j}^{(\mathrm{Lab})}=\mathcal{R}_{i k} \mathcal{R}_{j l} A_{k l}^{(\mathrm{Sun})}$, with $\mathcal{R}_{i j}$ representing merely a spatial rotation

$$
\mathcal{R}_{i j}=\left(\begin{array}{ccc}
\cos \chi \cos \Omega t & \cos \chi \sin \Omega t & -\sin \chi \\
-\sin \Omega t & \cos \Omega t & 0 \\
\sin \chi \cos \Omega t & \sin \chi \sin \Omega t & \cos \chi
\end{array}\right) .
$$

The Earth-based RF has an axis $x, y$, and $z$, while the Sunbased RF has $X, Y$, and $Z$ as an axis. Hence, $A_{i j}^{(\mathrm{Lab})} \equiv A_{i j}^{(x, y, z)}$ and $A_{i j}^{(\text {Sun })} \equiv A_{i j}^{(X, Y, Z)}$. Furthermore, by definition, the $z$ axis matches the direction of the Earth's rotation axis and the $x$ axis points from the Earth's center to the Sun on the vernal equinox in 2000 — for more details, see Ref. [47].

According to the transformation law mentioned above, the time-averaged bound (37) is $\left\langle(\kappa)^{z x}\right\rangle^{(\mathrm{Lab})}=$ $\frac{1}{2}(\sin \chi \cos \chi)\left[\left(\kappa^{X X}+\kappa^{Y Y}-2 \kappa^{Z Z}\right)^{\text {Sun }}\right]$. Given that $\operatorname{Tr}(\kappa)=0$, it holds that $\left\langle(\kappa)^{z x}\right\rangle^{(\mathrm{Lab})}=-\frac{3}{2}(\sin \chi \cos \chi)\left[\left(\kappa^{Z Z}\right)^{(\text {Sun })}\right]$, so that the upper bound (37) now constrains one of the diagonal elements of the $\kappa$ matrix (in the Sun's RF),

$$
|\tilde{S} \cos \chi \sin \chi|\left|\kappa^{Z Z}\right| \lesssim 1.7 \times 10^{-13} e \mathrm{fm}^{3} .
$$

As one considers that $\tilde{S}$ has the same order of magnitude of the usual Schiff moment, $\tilde{S} \sim 10^{-12} e \mathrm{fm}^{3}$, the bounds (37) and (39) allow for major LV coefficients acting on the nucleus physics, $\left|\kappa^{Z Z}\right| \lesssim 0.17$, although without enhancing the atomic EDM - a possibility that certainly deserves more investigation.

Such a scenario is also interesting if one takes into account the presence of the intrinsic LV nuclear EDM, $\boldsymbol{d}_{\mathrm{ilv}}$, stemming from LV interactions. The estimates in the literature for the Schiff moment [18] do not account for the last term in Eq. (32) nor Eq. (35), which could amplify/ modify the total Schiff moment. First, it is necessary to make presumptions about the structure of this LV EDM piece, such as it not being parallel to the nuclear spin I. A first possibility is to consider that $\boldsymbol{d}_{\text {ilv }}$ points in a specific fixed direction in spacetime, given by the LV background under consideration, without relation to the nuclear spin, such as it occurs in some LV nonminimal coupling systems, in which the LV background is coupled directly to the field strength [48]. In this case, the intrinsic $\boldsymbol{d}_{\text {ilv }}$ would not cause spin precession around the magnetic field, since it would not be written in terms of the spin I. Furthermore, the implied torque associated to the fixed direction of $\boldsymbol{d}_{\mathrm{ilv}}$ in spacetime could yield dissipation of an initial precession. In this case, the rate of the dissipation or change in the precession frequency would work as a channel for constraining the $d_{\mathrm{ilv}}$ magnitude. It is worth mentioning that such a spin-independent EDM (or MDM) in the direction of background does not appear in any nonminimal coupling, 
as the $C P T$-even nonminimal couplings $[40,41]$, for instance. As a second possibility, the EDM $\boldsymbol{d}_{\mathrm{ilv}}$ can also emerge as the result of the presence of LV coefficients in the nuclear interactions, properly coupled to the nuclear fields (instead of electromagnetic field). In this case, it is expected that $\boldsymbol{d}_{\mathrm{ilv}}$ should depend on both the spin $\mathbf{I}$ and background fields directions, so that the intrinsic LV nuclear EDM, $\boldsymbol{d}_{\mathrm{ilv}}$, could also imply precession, being subject to constraining by the procedure that led to the bound (37).

Considering the possibility of separating the repercussions of parity-odd and parity-even LV coefficients, we could choose to work with the Schiff moment (35) in order to focus on the $\boldsymbol{d}_{\text {ilv }}$ effects. Taking the nuclear spin aligned with the magnetic field in the $z$ axis, the usual Schiff moment does need to be considered for precession respects, so that the effective Schiff moment $S_{(3)}^{k}=\left(d_{\mathrm{ilv}}^{k} / 6\right) \int r^{2} \rho_{0}(r) d^{3} r$. Taking $\boldsymbol{d}_{\mathrm{ilv}}$ and $\boldsymbol{d}_{\delta}$ proportional but not parallel, $\left|\boldsymbol{d}_{\mathrm{ilv}}\right|=\beta\left|\boldsymbol{d}_{\delta}\right|$, for a constant $\beta$, it holds that $\boldsymbol{d}_{\mathrm{ilv}}^{k} \int r^{2} \rho_{0}(\boldsymbol{r}) d^{3} r=\beta \eta \tilde{S} \hat{k}$. Here, it was used that the piece $\left|\boldsymbol{d}_{\delta}\right| \int r^{2} \rho_{0}(\boldsymbol{r}) d^{3} r$ has the same order of magnitude of the Schiff moment $\tilde{S}^{k},\left|\boldsymbol{d}_{\delta}\right| \int r^{2} \rho_{0}(\boldsymbol{r}) d^{3} r \sim \eta \tilde{S}$. With this hypothesis, we obtain $\mathbb{S}_{(3)}^{k}=\beta \tilde{S} \hat{\boldsymbol{d}}_{\mathrm{ilv}}^{k} / 6$, with $(\eta \sim 1)$. In this case, if the vector $\boldsymbol{d}_{\text {ilv }}$ has an $x$ component, it turns out that $\mathbb{S}_{(3)}^{x}=\beta \tilde{S} / 6$. Following the route that led to bound (37), one achieves $\beta / 6 \lesssim 0.17$ or $\beta \lesssim 1.0$. The attainment of a non-null $\beta$, compatible with the current experimental measurements, can indicate the existence of nuclear intrinsic EDM (due to LV effects only), $\boldsymbol{d}_{\mathrm{ilv}}$, and sensitive Lorentz violation in nuclear systems. This is a new issue that remains to be properly investigated and involves the uncovering of the theoretical structure of $\boldsymbol{d}_{\mathrm{ilv}}$.

\section{ACKNOWLEDGMENTS}

The authors are grateful to CNPq, CAPES, and FAPEMA (Brazilian research agencies) for the financial support.
[1] W. Bernreuther and M. Suzuki, Rev. Mod. Phys. 63, 313 (1991); V. F. Dmitriev and I. B. Khriplovich, Phys. Rep. 391, 243 (2004).

[2] J. S. M. Ginges and V. V. Flambaum, Phys. Rep. 397, 63 (2004); Phys. Rev. A 65, 032113 (2002); J. Jesus and J. Engel, Phys. Rev. C 72, 045503 (2005).

[3] B. Lee Roberts and W. J. Marciano, Lepton Dipole Moments, Advanced Series on Directions in High Energy Physics (Word Scientific, Singapore, 2010).

[4] K. Jungmann, Ann. Phys. (Berlin) 525, 550 (2013); N. Yamanaka, Int. J. Mod. Phys. A 26, 1730002 (2017); J. Engel, M. J. Ramsey-Musolf, and U. van Kolck, Prog. Part. Nucl. Phys. 71, 21 (2013).

[5] A. D. Sakharov, Pis'ma Zh. Eksp. Teor. Fiz. 5, 32 (1967) [JETP Lett. 5, 24 (1967)].

[6] R. D. Peccei and H. R. Quinn, Phys. Rev. Lett. 38, 1440 (1977).

[7] C. T. Hill, Phys. Rev. D 91, 111702 (2015); 93, 025007 (2016); V. V. Flambaum, B. M. Roberts, and Y. V. Stadnik, Phys. Rev. D 95, 058701 (2017); C. T. Hill, Phys. Rev. D 95, 058702 (2017).

[8] W. C. Griffith, M. D. Swallows, T. H. Loftus, M. V. Romalis, B. R. Heckel, and E. N. Fortson, Phys. Rev. Lett. 102, 101601 (2009).B. Graner, Y. Chen, E. G. Lindahl, and B. R. Heckel, Phys. Rev. Lett. 116, 161601 (2017).

[9] I. I. Schiff, Phys. Rev. 132, 2194 (1963).

[10] O. P. Sushkov, V. V. Flambaum, and I. B. Khriplovich, Zh. Eksp. Teor. Fiz. 87, 1521 (1984) [Sov. Phys. JETP 60, 873 (1984)]; V. V. Flambaum, I. B. Khriplovich, and O.P. Sushkov, Nucl. Phys. A449, 750 (1986).

[11] R. A. Sen'kov, N. Auerbach, V. V. Flambaum, and V. G. Zelevinsky, Phys. Rev. A 77, 014101 (2008).
[12] V. A. Dzuba, V. V. Flambaum, and J. S. M. Ginges, Phys. Rev. A 61, 062509 (2000).

[13] V. V. Flambaum, Phys. Rev. A 60, R2611 (1999).

[14] J. Engel, J. L. Friar, and A. C. Hayes, Phys. Rev. C 61, 035502 (2000).

[15] V. V. Flambaum and A. Kozlov, Phys. Rev. C 85, 068502 (2012).

[16] V. V. Flambaum and J. S. M. Ginges, Phys. Rev. A 65, 032113 (2002).

[17] S. Ban, J. Dobaczewski, J. Engel, and A. Shukla, Phys. Rev. C 82, 015501 (2010).

[18] V. A. Dzuba, V. V. Flambaum, J. S. M. Ginges, and M. G. Kozlov, Phys. Rev. A 66, 012111 (2002).

[19] C.-P. Liu, M. J. Ramsey-Musolf, W. C. Haxton, R. G. E. Timmermans, and A.E. L. Dieperink, Phys. Rev. C 76, 035503 (2007).

[20] V. F. Dmitriev and R. A. Sen'kov, Phys. Rev. Lett. 91, 212303 (2003).

[21] N. Auerbach, V. V. Flambaum, and V. Spevak, Phys. Rev. Lett. 76, 4316 (1996); V. Spevak, N. Auerbach, and V. V. Flambaum, Phys. Rev. C 56, 1357 (1997).

[22] V. V. Flambaum and A. Kozlov, Phys. Rev. A 85, 022505 (2012).

[23] C.-P. Liu and J. Engel, Phys. Rev. C 76, 028501 (2007); J. Dobaczewski and J. Engel, Phys. Rev. Lett. 94, 232502 (2005); J. A. Ludlow and O. P. Sushkov, J. Phys. B46, 085001 (2013).N. Yamanaka, B. Sahoo, L. Yoshinaga, T. Sato, K. Asahi, and B. Das, Eur. Phys. J. A 53, 54 (2017).

[24] D. Colladay and V. A. Kostelecky, Phys. Rev. D 55, 6760 (1997); 58, 116002 (1998); S. R. Coleman and S. L. Glashow, Phys. Rev. D 59, 116008 (1999).

[25] V. A. Kostelecky and N. Russell, Rev. Mod. Phys. 83, 11 (2011). 
[26] S. M. Carroll, G. B. Field, and R. Jackiw, Phys. Rev. D 41, 1231 (1990); C. Adam and F. R. Klinkhamer, Nucl. Phys. B607, 247 (2001); B657, 214 (2003); Y. M. P. Gomes and P. C. Malta, Phys. Rev. D 94, 025031 (2016).

[27] V. A. Kostelecky and M. Mewes, Phys. Rev. Lett. 87, 251304 (2001); Phys. Rev. D 66, 056005 (2002); Phys. Rev. Lett. 97, 140401 (2006).

[28] C. A. Escobar and M. A. G. Garcia, Phys. Rev. D 92, 025034 (2015); A. Martín-Ruiz and C. A. Escobar, Phys. Rev. D 94, 076010 (2016).

[29] Q. G. Bailey and V. A. Kostelecky, Phys. Rev. D 70, 076006 (2004).

[30] R. Casana, M. M. Ferreira, Jr., and C. E. H. Santos, Phys. Rev. D 78, 105014 (2008).

[31] R. Casana, M. M. Ferreira, Jr., A. R. Gomes, and Paulo R. D. Pinheiro, Eur. Phys. J. C 62, 573 (2009).

[32] J. P. Noordmans, H. W. Wilschut, and R. G. E. Timmermans, Phys. Rev. C 87, 055502 (2013); Phys. Rev. Lett. 111, 171601 (2013); K. K. Vos, H. W. Wilschut, and R. G. E. Timmermans, Phys. Rev. C 92, 052501(R) (2015); Rev. Mod. Phys. 87, 1483 (2015).

[33] Y. V. Stadnik and V. V. Flambaum, Eur. Phys. J. C 75, 110 (2015).

[34] V. V. Flambaum and M. V. Romalis, Phys. Rev. Lett. 118, 142501 (2017).

[35] A. H. Gomes, V. A. Kostelecky, and A. J. Vargas, Phys. Rev. D 90, 076009 (2014).

[36] I. Altarev et al., Europhys. Lett. 92, 51001 (2010).

[37] M. Haghighat, I. Motie, and Z. Rezaei, Int. J. Mod. Phys. A 28, 1350115 (2013); S. Aghababaei, M. Haghighat, and I. Motie, Phys. Rev. D 96, 115028 (2017).
[38] Y. M. P. Gomes and P. C. Malta, Phys. Rev. D 94, 025031 (2016).

[39] A. F. Santos and F. C. Khanna, Phys. Rev. D 95, 125012 (2017).

[40] R. Casana, M. M. Ferreira, Jr., E. Passos, F. E. P. dos Santos, and E. O. Silva, Phys. Rev. D 87, 047701 (2013).

[41] J. B. Araujo, R. Casana, and M. M. Ferreira, Jr., Phys. Rev. D 92, 025049 (2015); Phys. Lett. B 760, 302 (2016).

[42] Y. Ding and V. A. Kostelecký, Phys. Rev. D 94, 056008 (2016).

[43] V. A. Kostelecky and M. Mewes, Phys. Rev. D 80, 015020 (2009); 88, 096006 (2013); M. Mewes, Phys. Rev. D 85, 116012 (2012).

[44] M. Schreck, Phys. Rev. D 89, 105019 (2014); 90, 085025 (2014); J. A. A. S. Reis and M. Schreck, Phys. Rev. D 95, 075016 (2017).

[45] R. C. Myers and M. Pospelov, Phys. Rev. Lett. 90, 211601 (2003); C. M. Reyes, L. F. Urrutia, and J. D. Vergara, Phys. Rev. D 78, 125011 (2008); Phys. Lett. B 675, 336 (2009); C.M. Reyes, Phys. Rev. D 82, 125036 (2010); 80, 105008 (2009).

[46] M. Pospelov and A. Ritz, Ann. Phys. (Amsterdam) 318, 119 (2005); P. A. Bolokhov, M. Pospelov, and M. Romalis, Phys. Rev. D 78, 057702 (2008).

[47] R. Bluhm, V. Alan Kostelecky, C. D. Lane, and N. Russel, Phys. Rev. Lett. 88, 090801 (2002); Phys. Rev. D 68, 125008 (2003); V. A. Kostelecky and M. Mewes, Phys. Rev. D 66, 056005 (2002).

[48] H. Belich, T. Costa-Soares, M. M. Ferreira, Jr., and J. A. Helayel-Neto, Eur. Phys. J. C 41, 421 (2005); H. Belich, L. P. Colatto, T. Costa-Soares, J. A. Helayel-Neto, and M. T. D. Orlando, Eur. Phys. J. C 62, 425 (2009). 